\title{
Influencia de los Parámetros de Carga en la Estabilidad Direccional de un Vehículo Combinado
}

\author{
Juan C. Horta ${ }^{1}$ y Antônio C. Canale ${ }^{2}$ \\ (1) Universidade Federal de Minas Gerais, Escola de Engenharia, Departamento de Engenharia \\ Mecânica, Av. Antônio Carlos 6.627, Campus Pampulha, 31270-901 Belo Horizonte, MG-Brasil \\ (e-mail: horta@demec.ufmg.br) \\ (2) Universidade de São Paulo, Escola de Engenharia de São Carlos, Depto. de Engenharia de \\ Materiais Aeronáutica e Automobilística, Av. Trabalhador Sãocarlense 400, Centro, \\ 13560-970 São Carlos, SP-Brasil (e-mail: canale@sc.usp.br)
}

\begin{abstract}
Resumen
En este trabajo se estudia la estabilidad direccional de un vehículo comercial combinado camióntractor semi-remolque (muy utilizado en Brasil), como función de los parámetros de carga. Para diferentes posiciones del centro de gravedad del conjunto semi-remolque-carga, se obtuvo las curvas que representan los movimientos característicos del vehículo en respuesta a la acción de una perturbación externa. Se calculó también, los factores de amortiguación para estos movimientos durante trayectorias curvas y rectas. Se estudió la influencia de la variación de la carga y del momento de inercia del semi-remolque para las diferentes posiciones del centro de gravedad adoptadas. Los resultados indican cambios significativos en la respuesta dinámica del vehículo con la variación de los parámetros de carga. Se determinó así las condiciones operacionales con menor margen de seguridad para que puedan ser evitadas por los usuarios. Se concluye que existen condiciones de carga que, aunque permitidas por la legislación vigente, deben ser evitadas.
\end{abstract}

\section{The Influence of Parameters of Load in the Directional Stability of Articulated Heavy Vehicle}

\begin{abstract}
This work is about the directional stability of truck-semitrailer vehicle, commonly used in Brazil, as a function of load parameters. For different positions of centre of gravity of semi-trailer, the curves that represent the characteristic movements and the ratio of damping for these movements during curved and straight trajectories are obtained. The influence of load and moment inertia variations for different adopted positions of the centre of gravity of semi-trailer was calculated. The results show significant changes on dynamic response of vehicle with the variations of load parameters. Thus, the operating conditions with less safety margins were determined, so they can be avoided by users. The study allows concluding that there are load conditions that although being accepted by the present legislation, must be avoided.
\end{abstract}

Keywords: articulated vehicles, tractor semi-trailer, vehicle dynamics, directional stability, heavy vehicles 


\section{INTRODUCCIÓN}

Los vehículos comerciales pesados combinados son ampliamente usados en todo el mundo y sobretodo en Brasil, donde el traslado de cargas mediante el transporte automotor llega a alcanzar más del $70 \%$ del peso total transportado (Estudos Técnicos NTC, 1996). Al mismo tiempo, se observa en Brasil un gran número de accidentes que involucra a este tipo de vehículos, causando grandes pérdidas materiales y humanas (Instituto de Pesquisa Econômica Aplicada, 2006). En la investigación de estos accidentes, se evidencia que un número significativo de ellos ocurre por la pérdida de la estabilidad direccional a velocidades de movimiento, que están dentro de los límites permitidos por la legislación, lo que es influenciado, principalmente, por el modo como es colocada la carga (Pflug, 1986).

Como normalmente los vehículos son homologados para las condiciones de vehículo vacío y con carga total, para las condiciones de cargas parciales, no se tiene una previsión de cual será el comportamiento dinámico del vehículo. El estudio de Fernández (1994), muestra que para algunas cargas parciales, los vehículos no cumplen con los requisitos de homologación. De este modo, son necesarios estudios que evalúen el comportamiento del vehículo en las condiciones de operación y carga más significativas, incluyendo las condiciones que corresponden a cargas parciales. En la literatura pueden ser encontrados varios estudios de este tipo (Fernández, 1994; Gutiérrez, 1999) aplicados a vehículos y condiciones de operación brasileñas.

El objetivo de este trabajo es el estudio teórico y analítico de la estabilidad direccional de un vehículo comercial pesado combinado muy utilizado en Brasil, considerando la influencia de las condiciones de carga, sobretodo en el caso de las cargas parciales más significativas. Para esto serán obtenidas las curvas que representan los movimientos característicos del vehículo en respuesta a la acción de una perturbación externa. Serán calculados también los factores de amortiguación para estos movimientos durante trayectorias curva y recta, y será evaluada la influencia de la variación de la carga y del momento de inercia del semi-remolque para las diferentes posiciones del centro de gravedad (c.g.) adoptadas. La estabilidad direccional será estudiada utilizando el método de cálculo y evaluación de las raíces del polinomio característico para una determinada condición de movimiento, que es obtenido a través de la solución complementaria de las ecuaciones de movimiento (Karnopp, 2004; Ellis, 1994). Como resultado podrán ser identificadas las condiciones operacionales y de cargamento con menor margen de seguridad para que puedan ser evitadas por los usuarios, posibilitando así el uso más eficiente y seguro del vehículo.

\section{METODOLOGIA}

El estudio es realizado teóricamente, a partir del modelo matemático de un vehículo combinado tipo camión-tractor semi-remolque, que presenta tres grados de libertad: deslizamiento lateral del camióntractor, rotación alrededor del eje vertical ("yaw") del camión-tractor y ángulo de rotación alrededor del eje vertical ("yaw") del semi-remolque. Son determinadas las ecuaciones del movimiento del vehículo en las direcciones correspondientes a dichos grados de libertad. Varias simplificaciones e hipótesis son adoptadas para hacer viable el análisis (Fernández, 1994; Gutiérrez, 1999), principalmente la hipótesis de las pequeñas perturbaciones que permite la linealidad de las ecuaciones. El movimiento del vehículo es asumido en la condición inicial de movimiento curvilíneo o rectilíneo en régimen permanente. La carga estática sobre cada eje es calculada a partir de la condición de equilibrio para el camión-tractor y para el semi-remolque (Fig. 1). También es calculada la variación de la carga vertical sobre las ruedas durante el movimiento en curvas.

El cálculo del coeficiente de rigidez lateral de los neumáticos es realizado considerando la influencia de la carga vertical y de varios parámetros del neumático (Pacejka, 2001). La fuerza lateral $(\mathrm{F} \psi)$ transmitida del pavimento al neumático puede entonces ser calculada como:

$\mathrm{F} \psi=\mathrm{N} \psi$

Donde $\mathrm{F} \psi \leq \mu \mathrm{fP}$, siendo que $\mathrm{N}$ es el coeficiente de rigidez lateral del neumático, $\mathrm{P}$ es la carga vertical sobre el neumático, $\psi$ es el ángulo de deslizamiento del neumático, y $\mu$ f es el coeficiente de adherencia efectivo entre el pavimento y el neumático. 


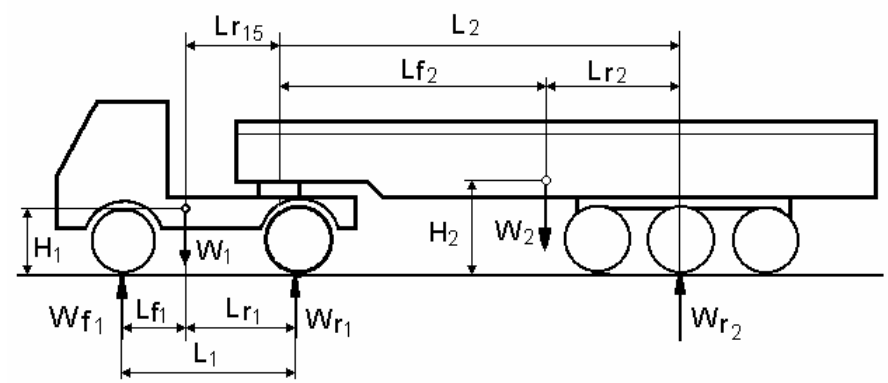

Fig. 1: Fuerzas estáticas que actúan sobre el vehículo.

Se adopta un procedimiento analítico para el estudio de la estabilidad direccional de la combinación vehicular, que consiste en el cálculo y evaluación de las raíces $(\lambda)$ del polinomio característico para una determinada condición de movimiento y de velocidad (V), que es obtenido a través de la solución complementaria de las ecuaciones de movimiento (Kuo, 1985; Dixon, 1996). Este procedimiento es conocido y muy utilizado en estudios de sistemas de control automático; se utiliza aquí por su mayor simplicidad en comparación con el método del Criterio de Routh- Hurtwitz (Vlk, 1982). También porque ofrece una información más completa sobre el sistema dinámico. Otros métodos, como por ejemplo el Criterio de Lyapunov y el Criterio de Nyquist, son más complejos y menos utilizados en este tipo de estudio, de acuerdo a lo indicado por Gutiérrez (1999). Así, son calculados los coeficientes del polinomio característico de cuarto orden $\left(\mathrm{K}_{4}, \mathrm{~K}_{3}, \mathrm{~K}_{2}, \mathrm{~K}_{1}, \mathrm{~K}_{0}\right)$, dados en la ecuación siguiente:

$$
\mathrm{K}_{4}(\mathrm{~V} \lambda)^{4}+\mathrm{K}_{3}(\mathrm{~V} \lambda)^{3}+\mathrm{K}_{2}(\mathrm{~V} \lambda)^{2}+\mathrm{K}_{1}(\mathrm{~V} \lambda)+\mathrm{K}_{0}=0
$$

Con ayuda de un programa computacional, es realizado el cálculo de los cuatro valores de las raíces del polinomio $(\lambda)$, lo que determina el carácter de la solución final. Considerando que todos los coeficientes del polinomio característico sean números reales, las raíces $(\lambda)$ pueden ser valores reales o resultar en pares complejos, con valores real e imaginario al mismo tiempo. La condición para considerar al vehículo como dinámicamente estable, será aquella cuando las raíces reales sean negativas o cuando el par complejo tenga la parte real negativa. Si estos valores fueren positivos el vehículo será considerado como inestable pudiendo tener respuesta exponencial u oscilatoria divergente ante una determinada perturbación externa (fuerzas externas transitorias, ráfaga de viento, irregularidades del pavimento, etc.). Los factores de amortiguación y la frecuencia amortiguada correspondientes a cada movimiento característico del vehículo son también calculados y brindan información sobre la respuesta del vehículo. Como la parte real de las raíces del polinomio representa el factor de amortiguación asociado con la atenuación de la amplitud $(\xi)$, y la parte imaginaria es la frecuencia amortiguada de la oscilación $(\eta)$, se pueden encontrar los factores de amortiguamiento de acuerdo con la siguiente ecuación:

$$
\zeta=\frac{1}{\sqrt{1+\left(\frac{\eta}{\xi}\right)^{2}}}
$$

Para el estudio fue seleccionado un conjunto camión-tractor con semi-remolque, muy utilizado en Brasil, compuesto por un camión-tractor LS 1941 de la Mercedes Benz de Brasil y un semi-remolque FNV - CARGA SECA del fabricante FNV. Una vez conocidos los valores del peso y del momento de inercia para el conjunto totalmente cargado y para el conjunto con el semi-remolque vacío (SR vacío), son determinados los valores de estas variables para las condiciones de carga (peso, $\mathrm{P}$ ), correspondientes a 0, 25, 50, 75 y $100 \%$ de la capacidad de carga total del semi-remolque. Las raíces del polinomio característico son entonces calculadas para el movimiento del vehículo durante la realización de una curva de radio $(\mathrm{R})$ igual a 152 metros, aumentándose la velocidad del movimiento hasta $240 \mathrm{~km} / \mathrm{h}$. También la simulación es hecha para el movimiento en dirección recta y hasta la misma velocidad indicada. En una segunda etapa son determinadas cinco posiciones del centro de gravedad (c.g.) longitudinal, siempre garantizándose el equilibrio estático del vehículo. 
Estas posiciones son estaciones de carga o posiciones que pueden coincidir con el centro de gravedad de cargas parciales colocadas en el semi-remolque en estas posiciones, de acuerdo a lo indicado en Pflug (1986) y Fernandes (1994). En la Fig. 2 son mostradas las posiciones más representativas para este estudio. Para cada una de estas posiciones se calculan las raíces del polinomio característico, para las mismas condiciones de movimiento que fueron explicadas anteriormente. Por último, a través de cambios sistemáticos en el peso y en el momento de inercia de giro ("yaw"), es investigada la influencia de estos dos factores en el comportamiento dinámico lateral y direccional.

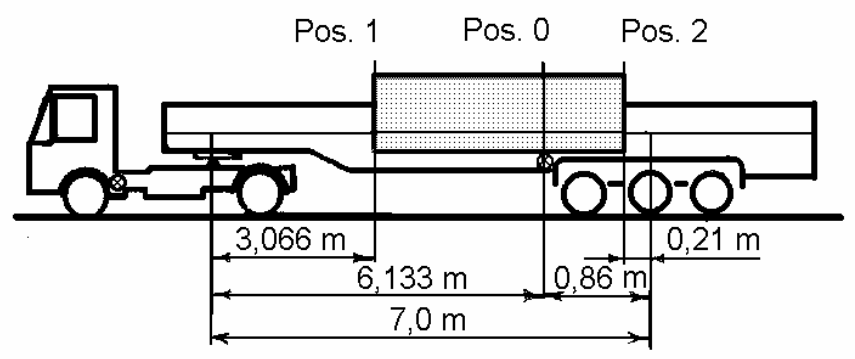

Fig. 2: Posiciones principales asumidas del centro de gravedad de las cargas parciales.

\section{RESULTADOS Y DISCUSIÓN}

En la Fig. 3 se observa que el aumento de la carga y del momento de inercia del semi-remolque, ocasiona la disminución del valor de la velocidad a partir de la cual el movimiento del vehículo se torna inestable. Así, para el caso del vehículo totalmente cargado se aprecia que, hasta una velocidad de $35 \mathrm{~km} / \mathrm{h}$ la respuesta del vehículo es exponencialmente convergente; de aquí y hasta $45 \mathrm{~km} / \mathrm{h}$ es una suma de dos movimientos exponenciales y uno oscilatorio, todos convergentes; a partir de 45 y hasta $145 \mathrm{~km} / \mathrm{h}$ es la suma de dos movimientos oscilatorios convergentes. A partir de ese último valor, se tendrá una raíz positiva y la posibilidad de pérdida de la estabilidad. Análisis semejantes fueron hechos para condiciones de carga parcial y sin carga, observándose la tendencia al mejoramiento del desempeño con la disminución del peso y del momento de inercia correspondiente. Sin embargo, estos resultados sufren variación cuando la posición del c.g. (en el plano longitudinal) cambia, como se puede observar en la Fig. 4. Aquí son mostradas solamente las raíces asociadas a los movimientos de respuesta del semi-remolque, porque ellas se tornan negativas primero que las otras. Los resultados muestran que con la posición del c.g. adelantada (posición 1) el vehículo en la curva va a perder la estabilidad a una velocidad próxima de la velocidad de vuelco $(90 \mathrm{Km} / \mathrm{h})$, mientras que con el c.g. en la posición 0 el vehículo se volcará antes de alcanzar el valor de la velocidad crítica (valor de velocidad de perdida de la estabilidad). Esto es observado también en la recta, donde la velocidad crítica para la posición 1 es de $130 \mathrm{~km} / \mathrm{h}$ aproximadamente, velocidad esta que es frecuente en las autopistas brasileñas. Para la posición "0" no se observan valores positivos de la parte real del par complejo en la recta, pero para la posición 2 el comportamiento del vehículo se afecta, lo que es evidenciado por el menor valor de la velocidad crítica en curva $(80 \mathrm{~km} / \mathrm{h})$, que es casi el mismo para el movimiento en la recta. Así, la pérdida de estabilidad puede ocurrir a velocidades que están dentro de los límites permitidos, lo que aumenta la probabilidad de accidentes ante cualquier perturbación externa, inclusive en la recta. Hasta para el caso de cargas parciales muy bajas el comportamiento dinámico se mostró muy perjudicado con la variación de la posición longitudinal del c.g.

Con relación a los factores de amortiguamiento, se evidenció que para la posición 2 del c.g. ellos llegan a ser iguales a cero en las velocidades de perdida de la estabilidad, hasta en el caso de movimiento en recta. Para la posición adelantada del c.g. (posición 1) los valores llegan a ser bajos lo que significa movimientos de respuesta combinados con bajo margen de seguridad. En este último caso el vehículo es estable, pero demorará más en volver a la condición de movimiento inicial (antes de la perturbación). También pudo ser constatado que la influencia del peso en el comportamiento dinámico del vehículo es menor que la influencia del momento de inercia de giro ("yaw"). El aumento de este último parámetro compromete mucho más la estabilidad dinámica del vehículo. 


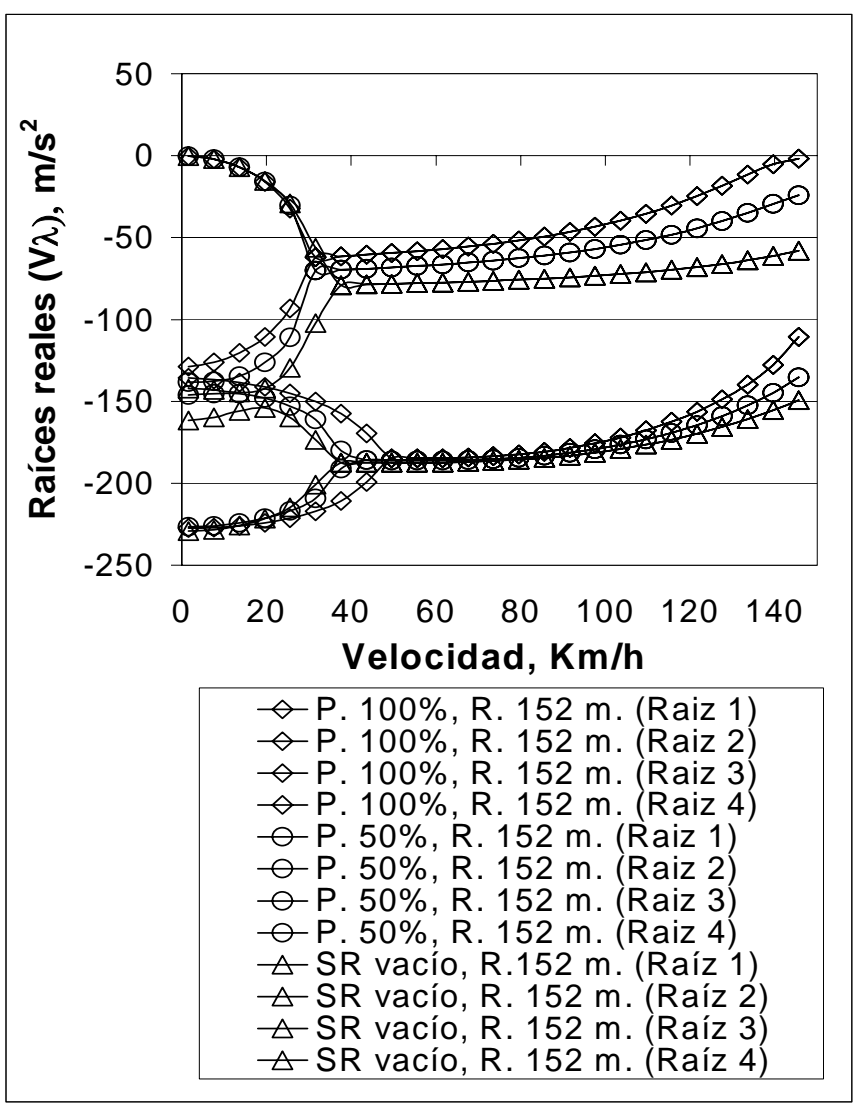

Fig. 3: Raíces reales para diferentes cargas del semi-remolque y en movimiento en curva.

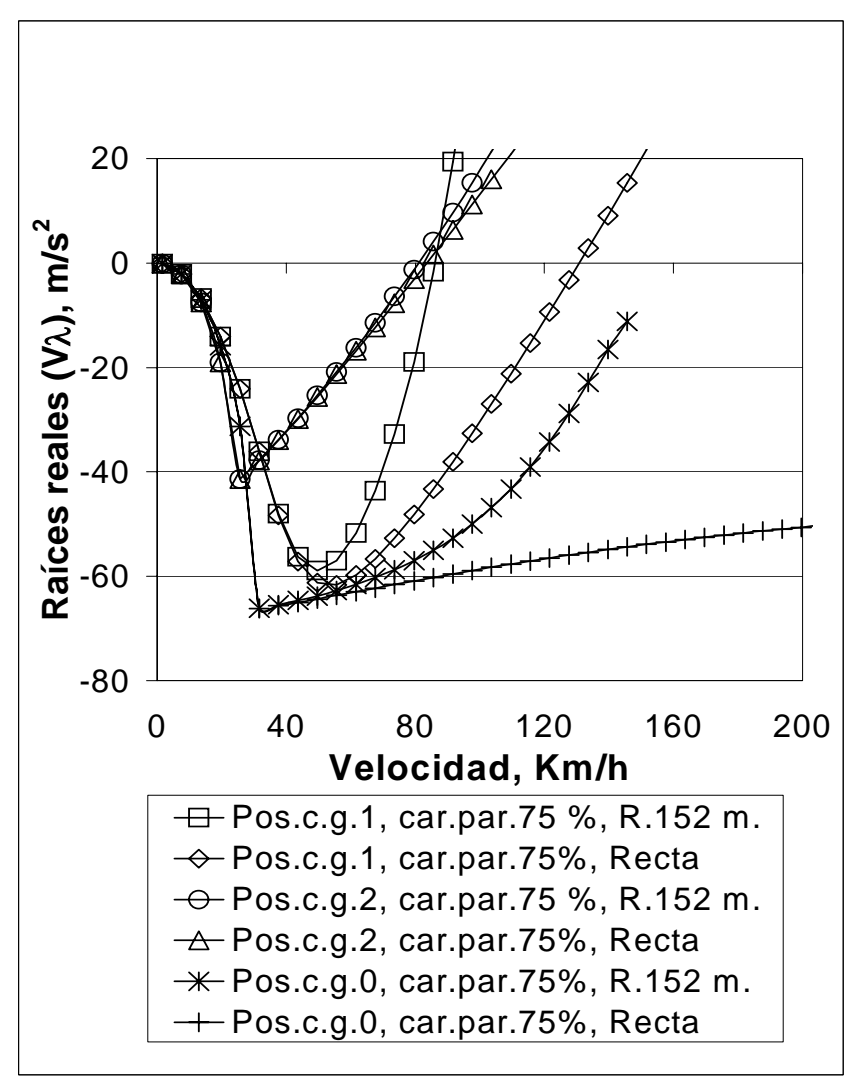

Fig. 4: Raíces reales para las posiciones del c.g. en el plano longitudinal de una carga parcial equivalente al $75 \%$ de la carga total y para el movimiento del vehículo en curva y recta. 


\section{CONCLUSIONES}

De los resultados mostrados y su análisis se puede concluir lo siguiente: 1) los usuarios de este tipo de vehículo no deben sobrecargarlo (lo que es practicado comúnmente en Brasil) para no disminuir el margen de movimiento seguro; 2) colocar las cargas en la posición "2", próximas de los ejes del semi-remolque (lo que es común en Brasil para pasar sin problemas en las balanzas de control de peso por ejes situadas en algunas autopistas brasileñas), compromete mucho el comportamiento dinámico del vehículo; 3) las cargas deberán colocarse de tal modo que la posición del c.g. del conjunto semi-remolque - carga quede próxima de la posición del c.g cuando el vehículo está vacío, ya que también en la posición adelantada del c.g. (posición "1") se observa un comportamiento dinámico desfavorable; 4) deben respetarse rigurosamente los límites de velocidad especiales y mas restrictos para este tipo de vehículos, ya que fueron observadas condiciones de movimiento con probabilidad de respuesta dinámica inestable para valores de velocidad que son permitidos normalmente en las autopistas brasileñas; 5) el análisis realizado muestra claramente la necesidad de evaluar los vehículos, considerando todas las condiciones operacionales posibles, ya que, como fue mostrado, existen condiciones que corresponden a cargas parciales en que el vehículo, que tienen un desempeño mucho mas desfavorable, que para el vehículo con carga total o vacío.

\section{REFERENCIAS}

Dixon, J.C.; Tires, suspension and handling, $2^{\text {nd }}$ edition. SAE, Warrendale - USA (1996).

Ellis, J.R.; Vehicle handling dynamics, Page Broos, London - UK (1994).

Estudos técnicos NTC Bridgestone Firestone; - Lei da balança no Brasil e no mundo -1, Transporte Moderno: (378), Suplemento (1996).

Fernandes, D.L.; Estudo da freagem de autoveículos rodoviários articulados pesados, Dissertação de Mestrado. Escola de Engenharia de São Carlos, Universidade de São Paulo, São Carlos, Brasil (1994).

Gutiérrez, J.C.; Estudo da estabilidade direcional de um veículo combinado cavalo semi-reboque, Tese de Doutorado. Escola de Engenharia de São Carlos, Universidade de São Paulo, São Carlos, Brasil (1999).

Instituto de Pesquisa Econômica Aplicada; Impactos Sócias e Econômicos dos Acidentes de Trânsito nas Rodovias Brasileiras. [SI], Dezembro (2006).

Karnoop, D.; Vehicle Stability, Marcel Dekker, Inc., Course of Lecture, University of California -USA (2004).

Kuo, B.C.; Sistemas de Controle Automático, 4 ed., Rio de Janeiro, Prentice-Hall (1985).

Pacejka, H.B.; Tire and Vehicle Handling. $1^{\text {st }}$ edition. SAE, Warrendale - USA (2001).

Pflug, H.C.; Lateral dynamic behaviour of truck-trailer combinations due to the influence of the load, Vehicle System Dynamics: 15, 155-175 (1986).

Vlk, F.; Lateral dynamics of commercial vehicle combination - a literature survey, Vehicle System Dynamics: 11(6), 305-324 (1982). 